\title{
Synthesis and post-treatments of biomimetic apatites: How working conditions may configure final physico-chemical features
}

\author{
Christophe Drouet ${ }^{\mathrm{a}}$, Nicolas Vandecandelaere and Christian Rey \\ CIRIMAT Carnot Institute, University of Toulouse, UMR CNRSIINPTIUPS 5085, Toulouse, France
}

\begin{abstract}
Nanocrystalline apatites constitute the mineral part of hard tissues, and can be reproduced synthetically. Nonetheless, the impact of synthesis/post-synthesis parameters is often disregarded. Based on actualized knowledge on their physico-chemical features, we investigated these aspects on a systematic experimental basis. The apatite maturation state has a direct effect on the surface and core of the nanocrystals. Drying and re-immersion aspects were also examined in view of applications as implantable biomaterials: an equilibration of the samples surface is proposed to avoid acidification phenomena after re-immersion of dried samples.
\end{abstract}

\section{INTRODUCTION}

Calcium phosphate nanocrystalline apatites are poorlycrystallized compounds. They are nonstoichiometric and the nanocrystals exhibit a non-apatitic surface layer containing labile ionic species (non-apatitic ionic environments) (Eichert et al. 2008; Gomez-Morales et al. 2013): this layer is thought to play a key role on surface/interfacial reactivity (ion exchange mechanisms, adsorption/desorption of (bio)molecules,...) due to the labile character of its constitutive ions. Although the preparation of nanocrystalline apatites has been the object of various studies, the impact of synthesis and postsynthesis conditions is seldom investigated in depth. In this paper, we point out via a systematic study the role of synthesis parameters (maturation time in solution, nature of counter-ions, temperature, $\mathrm{pH}$ ) on apatite nanocrystals features. We also investigated the effects of post-synthesis treatments (re-immersion in solution, surface equilibration, thermal treatment). This work may prove helpful for designing production lines for biomimetic apatites.

\section{MATERIALS AND METHODS}

Nanocrystalline apatites were prepared by precipitation in aqueous medium. After a pre-selected maturation time, the precipitate was filtered, washed and freeze-dried. XRD (Seifert XRD 3000 TT diffractometer, $\mathrm{Cu}_{\mathrm{Ka} 1 \mathrm{Ka} 2}$, FTIR (Nicolet 5700 spectrometer) and chemical analyses were used to determine the structural and crystallographic features of the samples obtained.

\section{RESULTS AND DISCUSSION}

\section{Effect of synthesis conditions}

XRD data indicated an improvement in pattern resolution for increasing maturation times in the precipitating

\footnotetext{
e-mail: christophe.drouet@ensiacet.fr
}

medium. Profile-fitting analyses (JANA software) pointed out an increase in crystallite size (e.g. mean lengths varying from 9.5 to $20.4 \mathrm{~nm}$ upon maturation between 20 min and 20 days) accompanied by a decrease in crystal disorder (as derived from the Vogel and Hosemann (1970) method). TEM analyses confirmed these observations and indicated that the plate-like morphology - typical of biomimetic apatites - was conserved independently of the maturation time tested. Chemical titrations of calcium and orthophosphate ions indicated that this modification of crystallographic features was accompanied by an increase in overall $\mathrm{Ca} / \mathrm{P}$ mole ratio $1.30 \pm 0.02$ and $1.48 \pm 0.02$ for the same maturation time range. These values, noticeably lower than 1.67 found for stoichiometric hydroxyapatite, unveil the nonstoichiometric character of such samples, despite a progression towards stoichiometry upon maturation in solution. FTIR data confirmed this point by showing a progressive increase in $\mathrm{OH}^{-}$ band intensity (e.g. libration band at $631 \mathrm{~cm}^{-1}$ ) and a simultaneous decrease in the intensity of the bands associated to non-apatitic (surface) environments (e.g. non-apatitic $\mathrm{HPO}_{4}^{2-}$ band at $\left.530 \mathrm{~cm}^{-1}\right)$. All of these results illustrate the strong impact of the initial maturation time on the characteristics of the apatite phase. These effects are especially pronounced during the first hours after precipitation and up to 3 days (beyond which a much slower evolution is seen).

Additional maturation tests were undergone but with a modification of maturation temperature $\left(37^{\circ} \mathrm{C}, 50^{\circ} \mathrm{C}\right.$ or $100^{\circ} \mathrm{C}$ ). Temperature was then found to have similar effects as maturation time, as a significant progression in maturation state was noticed in this temperature range, probably indicative of faster crystal growth (acceleration of maturation process leading to chemical compositions closer to stoichiometry). These findings should be fully considered when intending to produce (e.g. at an industrial scale) or test biomimetic apatites.

The effect of a change in synthesis $\mathrm{pH}$ was also followed (range 6-11, et RT); a pH increase being linked to an increase in supersaturation of the medium relatively to apatite (increase of the relative contents in $\mathrm{PO}_{4}^{3-}$ and $\mathrm{OH}^{-}$ ions, Elliott (1994)). At pH 6, brushite was precipitated rather than apatite. For the other $\mathrm{pH}$ values, apatite was 
the unique crystallized phase. FTIR showed that the amount of non-apatitic $\mathrm{HPO}_{4}^{2-}$ ions decreased while $\mathrm{OH}^{-}$ ions increased by raising the synthesis $\mathrm{pH}$ in this range. Samples carbonation (up to $3 \mathrm{wt} . \%$ ) was also noticed (bands in the range $1370-1550 \mathrm{~cm}^{-1}$ ) upon alkalinization of the medium; the overall $\mathrm{Ca} /(\mathrm{P}+\mathrm{C})$ content remained however essentially unchanged and the crystallinity state did not improve (carbonate is indeed a crystal growth inhibitor for apatite).

Different starting salts were used for additional synthesis tests. A replacement of ammonium by sodium in the phosphate starting salt was found to lead to a non-negligible incorporation of sodium in calcium sites (between 2 and $7 \mathrm{~mol} \mathrm{\%}$ in cationic sites, for maturation times of 20 min to 20 days), which was accompanied by a decrease in crystallinity (monovalent sodium substituting for bivalent calcium). These findings stress out the necessary caution to be taken when selecting starting salts to precipitate nanocrystalline apatite compounds. Indeed, a great number of counter-ions (exemplified here with sodium) are likely to be incorporated to some extent in the apatitic lattice, thus potentially modifying apatite behavior.

\section{Effect of selected post-treatments}

Bone-related bioceramics are intended to be used or tested in wet environments (e.g. in contact with body fluids, cell culture media...). However, they are generally employed by end-users in their dry state. Therefore, the question of a potential evolution during immersion or after selected pre-treatments prior to scaffold preparation (e.g. sterilization) may be reasonably raised.

Drying processes were found to affect the fine structuration of the surface of the nanocrystals, which was shown by FTIR spectral analysis versus drying time (progressive disappearance of fine spectral features, especially in the ${ }_{3} \mathrm{PO}_{4}$ domain). Other experiments were also run by immersing freeze-dried apatite samples in aqueous media (water or retrieved precipitating medium). FTIR data revealed that the dried samples underwent noticeable physico-chemical modifications after immersion, with an important decrease of the non-apatitic $\mathrm{HPO}_{4}^{2-}$ content and an increase of apatitic $\mathrm{OH}^{-}$. These findings may be related to modifications of the nanocrystals surface characteristics observed during the maturation process itself, and accelerated by the drying process. The drying stage leads to some surface amorphization, exposing more "disorganized" ions. After re-immersion in aqueous medium, the top-surface looselybound ions may then be more easily released thus "accelerating" the maturation process of apatite in humid conditions. The release of protonated phosphate ions (especially as $\mathrm{H}_{2} \mathrm{PO}_{4}^{2-}$ formed either by interaction between two surface $\mathrm{HPO}_{4}^{2-}$ ions or by reaction of $\mathrm{HPO}_{4}^{2-}$ with a water molecule) could explain the acidification of the medium, which was indeed experimentally verified either in water, in $\alpha$ MEM or in DMEM cell culture media (Fig. 1).

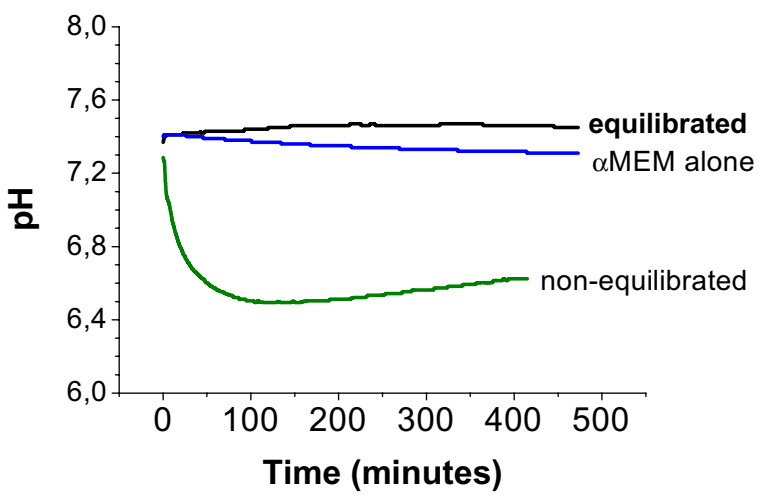

Figure 1. $\mathrm{pH}$ evolution after re-immersion of nanocrystalline apatite (1 day matur.) in $\alpha M E M$.

Such an acidification may however prove to be deleterious to the testing of apatite-based bioceramics cell activity (e.g. cell adhesion, proliferation, cytotoxicity...) and also possibly in vivo after implantation (although dynamic conditions are then provided). Therefore, attempts to solve this acidification issue, by way of an "equilibration" step, were investigated especially by washing the precipitate with an alkaline solution during the synthesis stage (between the filtration and the washing step, and thus prior to the freeze-drying stage). Our results indicated that washing with a solution of $\mathrm{Na}_{3} \mathrm{PO}_{4} \cdot 12 \mathrm{H}_{2} \mathrm{O}$ $(\mathrm{pH} 11)$ adequately allowed us to equilibrate the surface of the apatite nanocrystals so as to avoid any acidification after re-immersion in solution. This was verified by reproducing the above-mentioned immersions tests. This alkaline treatment may indeed modify the contents of the hydrated layer, e.g. by increasing the amount of $\mathrm{PO}_{4}^{3-}$ in relation to protonated phosphate ions, thus limiting efficiently a subsequent release of proton-containing ions upon re-immersion.

Apart from re-immersion, thermal treatments may be seen as another type of "post-treatment" interesting to consider when dealing with the production of nanocrystalline apatite compounds, for example in view of sterilization by heat or during sample cutting processes. We have thus investigated the effects of a thermal posttreatment of nanocrystalline apatite powders at varying temperatures (up to $100^{\circ} \mathrm{C}$ ) for 3 days. XRD data revealed that the apatite phase was maintained during these treatments and crystallite dimensions did not significantly vary. FTIR data however pointed out a decrease in nonapatitic $\mathrm{HPO}_{4}^{2-}$ content and in apatitic $\mathrm{OH}^{-}$as compared to the unheated sample. Also, water from the non-apatitic layer can also be evolved upon heating. These results underline a change in hydration state/composition of nanocrystalline apatites when subjected to heat treatments (for sterilization or during other processing steps). These alterations do not lead to a destruction of the apatite phase nor of nanocrystalline features; however their occurrence should be kept in mind as they are bound to modify the behavior of biomimetic apatite bioceramics, especially by altering their surface state which controls most interfacial phenomena. 
The authors thank the Agence Nationale de la Recherche for funding (PICF Biocapabili).

\section{References}

[1] D. Eichert, C. Drouet H. Sfihi, C. Rey, C. Combes, Biomaterials research advances. New York: Nova science publishers, 2008, 93-143.
[2] J.C. Elliott, Studies in inorganic chemistry vol 18, 1994, Amsterdam: Elsevier.

[3] J. Gomez-Morales, M. Iafisco, J.M. Delgado-Lopez, S. Sarda, C. Drouet, Prog. Cryst. Growth Charact Mater 59, 2013,1-46.

[4] W. Vogel, R. Hosemann, Evaluation of paracrystalline distortions from line broadening. Acta Cryst A 1970;26:272. 